\title{
Temperature dependence of the thermoelectric properties of Si doped SiC
}

\author{
Yoichi Okamoto, Hiroshi Inai and Jun Morimoto \\ Department of Materials Science and Engineering, National Defense Academy, 1-10-20 Hashirimizu, Yokosuka 239-8686.
}

Received April 23, 1998

\section{SYNOPSIS}

Thermoelectric properties of the $\mathrm{Si}$ doped $\mathrm{SiC}$ were measured. It is intended to reduce the thermal conductivity by addition of isoelectric element Si. Measurements of electrical resistivity, thermoelectric power and thermal conductivity were made on $\mathrm{SiC}$ thermoelectric semiconductor as a function of both Si doping concentration (over the range of 1.0 wt. $\% \sim 40.0$ wt.\%) and temperature (from room temperature to $750{ }^{\circ} \mathrm{C}$ ). Measurements of Hall coefficient, X-ray crystallography and EPMA were also made on these samples. The thermal conductivity decreases with increase of Si concentration. At Si concentration of $40.0 \mathrm{wt} . \%$, minimum thermal conductivity reached to $13 \mathrm{~W} / \mathrm{mK}$. The figure of merit $\mathrm{Z}$ was calculated from electrical resistivity, thermoelectric power and thermal conductivity. The maximum value of the figure of merit reaches $2 \times 10^{-4} \mathrm{~K}^{-1}$ at $750^{\circ} \mathrm{C}$ and Si concentration of $40.0 \mathrm{wt} . \%$.

KEY WORDS

thermoelectric material, figure of merit, $\mathrm{SiC}$

\section{Introduction}

Recently, strong effort has been directed towards the development of sintered thermoelectric materials ${ }^{1,2)}$ with improved characteristics at comparatively high temperature. The thermoelectric materials are evaluated by using the figure of merit $Z$. The figure of merit $Z$, which signifies the thermal to electrical energy conversion efficiency of the material, is defined by the following equation,

$$
\mathrm{Z}=\alpha^{2} / \rho \kappa
$$

where $\alpha, \rho$ and $\kappa$ are thermoelectric power, electrical resistivity and thermal conductivity, respectively. One of the criterion for the practical use of the thermoelectric materials can be expressed as,

$$
\mathrm{ZT} \geqq 1
$$

where $\mathrm{T}$ is the absolute temperature. It is difficult to decrease $\rho$ and $\kappa$, and to increase $\alpha$ simultaneously, because $\alpha$ and $\rho$ complexly depend on many parameters such as carrier concentration, carrier mobility, effective mass of carrier and etc. On the other hand, geometric factors (e. g. pores) also have effects both on $\rho$ and $\kappa$.

Recently, $\mathrm{SiC}$ interests some workers as a thermoelectric material ${ }^{3-5)} . \mathrm{SiC}$ is a wide gap semiconductor which has enough mechanical strength and chemical stability even in the high temperature region. Therefore it is expected to use in high temperature region. But this material has large electrical resistivity and large thermal conductivity. These properties are not favorable to increase the figure of merit. To reduce the large electrical resistivity, we have studied the thermoelectric properties of p-type $\mathrm{SiC}$ with doping of $\mathrm{Al}^{6)}, \mathrm{Ag}^{7}, \mathrm{Cu}^{8)}$ and $\mathrm{Ni}^{9)}$. These dopant atoms form effective acceptor levels in $\mathrm{SiC}$ and the electrical resistivity is reduced drastically.

In this paper, isoelectric element $\mathrm{Si}$ is selected as a dopant to reduce the thermal conductivity. By the addition of $\mathrm{Si}$, it is intended to introduce the scattering center of the thermal conduction.

\section{Experiments}

\subsection{Sample Preparation}

Sample preparation procedure is almost the same as described in our previous papers ${ }^{6-9)} . \beta-\mathrm{SiC}$ (average particle size $0.15 \mu \mathrm{m}$ and BET surface area $19.5 \mathrm{~m}^{2} / \mathrm{g}$, Mitsui Toatsu Co. Ltd., MSC-20) and Si (Kojundokagaku kenkyusyo Co. Ltd., $99.99 \%$ purity) were the starting materials. Slurries were made from mixed powders of $\mathrm{SiC}$ and $1.0 \sim 40.0 \mathrm{wt} . \%$ Si powders in polyethylene jars with nylon coated steel balls as the grinding media and ethanol solution as the mixing agent. After mixing for 20 hours, the slurries were dried. The dried mixture was passed through 100 mesh sieve, granulated using 50 mesh sieve and pressed into $20 \mathrm{~mm} \phi \times$ 
$6 \mathrm{~mm}$ pellet at $1 \times 10^{7} \mathrm{~N} / \mathrm{m}^{2}$. Then the pellets were sealed into an evacuated vinyl resin tube and then pressed isostatically at $2 \times 10^{8} \mathrm{~N} / \mathrm{m}^{2}$.

Each pellet was covered with the same compositional powder to prevent the compositional change during the sintering process and then placed into a carbon crucible. The sintering procedure was carried out in a furnace with a $\mathrm{RF}$ induction heater. It was heated up to $1000^{\circ} \mathrm{C}$ at a rate of $20^{\circ} \mathrm{C} / \mathrm{min}$. in vacuum, then $\mathrm{Ar}$ gas was introduced up to atmospheric pressure. Next the temperature was raised to the sintering temperature $\left(2100^{\circ} \mathrm{C}\right)$ at a rate of $10^{\circ} \mathrm{C} / \mathrm{min}$. and the sample was kept at this temperature for 2 hours and cooled naturally down to room temperature. Sintered materials were cut into rectangular shaped specimens of 3 $\times 4 \times 8 \sim 10 \mathrm{~mm}^{3}$ in dimensions.

\subsection{Sample Measurement}

The crystal structure and constituent of manufactured samples were studied by using powder X-ray diffractometer and EPMA (Electron Probe Micro Analyzer).

The measurements of thermoelectric power and electrical resistivity were ranging from room temperature to around $750{ }^{\circ} \mathrm{C}$. The measurements of thermal conductivity were ranging from room temperature to around $300{ }^{\circ} \mathrm{C}$. In the calculation of the figure of merit, the thermal conductivity was assumed to be constant above $300^{\circ} \mathrm{C}$.

Conventional DC method and conventional DC 4 probe method were employed for the measurements of thermoelectric power and electrical resistivity, respectively. The thermal conductivity measurement was made by a differential phase analysis of PPE (Photo Pyro Electric) method reported in our previous paper ${ }^{10,11)}$.

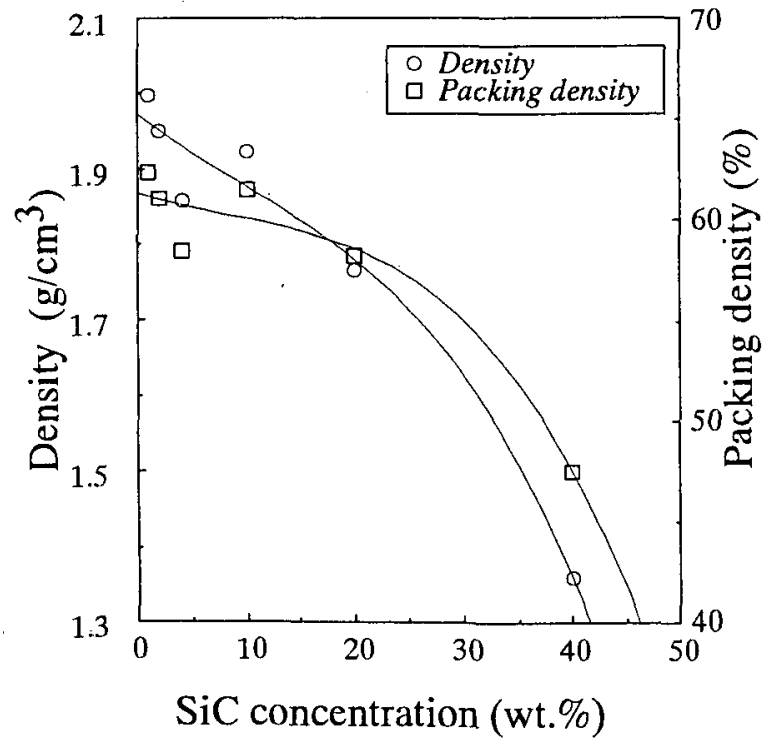

Fig.1 The Si concentration dependence of the density and packing density of the prepared sample.
In the PPE method one can measure thermal diffusivity $\alpha_{s}$. The thermal conductivity is given by

$$
\kappa=\alpha_{\mathrm{s}} \cdot \mathrm{d} \cdot \mathrm{C}
$$

here, $\kappa, \alpha_{\mathrm{s}}, \mathrm{d}$ and $\mathrm{C}$ are thermal conductivity, thermal diffusivity, density and specific heat, respectively. We calculated specific heat of $\mathrm{SiC} / \mathrm{Si}$ system from that of $\mathrm{SiC}$ and $\mathrm{Si}$. The values of $\mathrm{d}$ and $\alpha_{\mathrm{s}}$ were measured. At room temperature, we used pyro electric material $\mathrm{PVF}_{2}$ (Poly Vinyliden diFloride) films as heat sensor in the PPE measurement because of its high sensitivity. In the higher temperature region, we used thermocouple as mentioned in previous reports ${ }^{6-9)}$.

To clarify the carrier concentration, the Hall effects were measured by using ordinary van der Pauw method.

\section{Results and Discussion}

The addition of $\mathrm{Si}$ affects the crystalline structure of samples. The all X-ray diffraction peaks of the samples except $1.0 \mathrm{wt} . \%$ Si concentration were assigned to $3 \mathrm{C}-\mathrm{SiC}$ $(\beta$-SiC). Only X-ray diffraction profile of the sample with $1.0 \mathrm{wt} . \% \mathrm{Si}$ consists of both $3 \mathrm{C}-\mathrm{SiC}(\beta-\mathrm{SiC})$ and $6 \mathrm{H}-\mathrm{SiC}$ $(\alpha-\mathrm{SiC})$. At the sintering temperature of $2100{ }^{\circ} \mathrm{C}, \mathrm{SiC}$ transforms from $3 \mathrm{C}$ phase to $6 \mathrm{H}$ phase ${ }^{5,12)}$. According to the previous papers ${ }^{6.9)}$, all samples doped with $\mathrm{Al}, \mathrm{Ag}, \mathrm{Cu}$ and $\mathrm{Ni}$ which were sintered at $2100{ }^{\circ} \mathrm{C}$ show $6 \mathrm{H}$ phase. Addition of Si may have an effect to keep the $\mathrm{SiC}$ in $3 \mathrm{C}$ phase. No diffraction peak from Si was observed even from

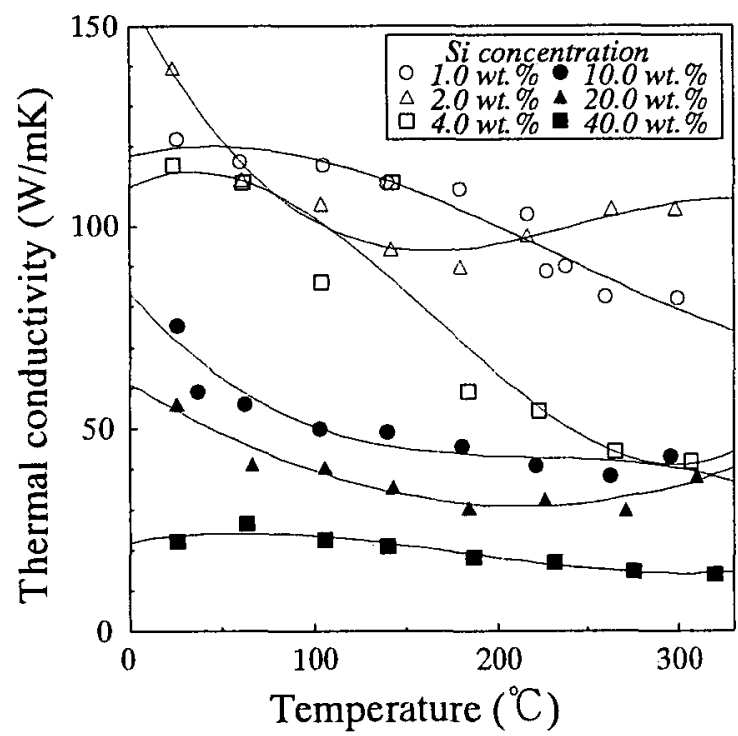

Fig.2 The temperature dependence of the thermal conductivity as a function of the Si concentration measured by using PPE method. In this figure, fig. 2 and fig. 3 , marks represent the measured points and solid lines represent the result of the polynomial which fitted to the experimental data for calculation of figure of merit. 
the sample of $40.0 \mathrm{wt} . \%$ Si concentration. No impurities were found from analysis by EPMA.

Figure 1 shows the Si concentration dependence of the density and packing density of the sintered samples. The right side axis of the ordinates means the packing density and the left side axis of the ordinates means the sintering density. Both density and packing density decrease with increase of $\mathrm{Si}$ concentration especially at higher $\mathrm{Si}$ concentration. The sample with $40.0 \mathrm{wt}$. $\% \mathrm{Si}$ concentration is the most porous sample in our sample preparation procedure $^{6-9)}$.

Figure 2 shows the temperature dependence of the thermal conductivity as a function of the Si concentration. All the samples show the weak decrease as temperature increases. Especially, the value of sample with $40.0 \mathrm{wt} . \%$ $\mathrm{Si}$ concentration is almost independent of temperature. At room temperature and at around $300{ }^{\circ} \mathrm{C}$, thermal conductivity decreases with increase of $\mathrm{Si}$ concentration. From this figure, minimum thermal conductivity decreased to $13 \mathrm{~W} / \mathrm{mK}$. We can say that addition of Si $40.0 \mathrm{wt} . \%$ decreases the thermal conductivity to the one tenth of the value for the $\mathrm{SiC}$ prepared by the same procedure without $\mathrm{Si}$. Thermal conductivity at room temperature decreases gradually with increase of Si concentration in the measured $\mathrm{Si}$ concentration range. On the other hand, the thermal conductivity at around $300{ }^{\circ} \mathrm{C}$ decreases steeply at lower $\mathrm{Si}$ concentration and decreases slowly at higher Si concentration range.

Figure 3 shows the temperature dependence of the electrical resistivity as a function of the Si concentration. The values of all samples decrease with increase of

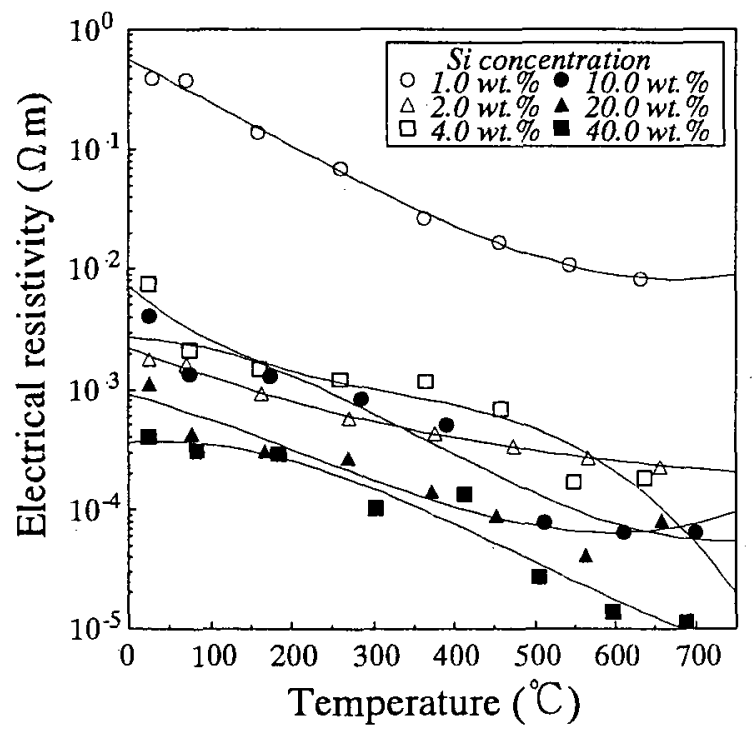

Fig.3 The temperature dependence of the electrical resistivity as a function of the Si concentration. temperature and more all samples have the similar gradient of the plots. At room temperature and at around $750{ }^{\circ} \mathrm{C}$, the electrical resistivity shows steep decrease at lower $\mathrm{Si}$ concentration and decreases slowly at higher $\mathrm{Si}$ concentration with increase of Si concentration.

From the Hall effects measurement, hole carrier concentration is calculated up to $10^{26} / \mathrm{m}^{3}$. All samples show the Hall voltage less than $40 \mathrm{nV}$ under the $7 \mathrm{kOe}$ magnetic field and $200 \mathrm{~mA}$ sample current. This large hole concentration is very close to the value of carrier concentration for metal. Since this SiC/Si system has typical semiconductor-like temperature dependence of electrical resistivity, this carrier concentration value may be too large.

Figure 4 shows the temperature dependence of the thermoelectric power as a function of the Si concentration. The all samples show the monotonous increase with increase of temperature. And more, all samples show the almost same gradients of the plots. At room temperature, the higher concentration samples show the lower thermoelectric power. On the other hand, at around 750 ${ }^{\circ} \mathrm{C}$, there are no obvious relation between the thermoelectric power and the Si concentration.

Figure 5 shows the temperature dependence of the figure of merit as a function of the Si concentration. At room temperature, the samples except $1.0 \mathrm{wt} . \%$ and $40.0 \mathrm{wt} . \%$ of Si concentration show almost same value of the figure of merit. On the other hand, at around $750{ }^{\circ} \mathrm{C}$, the figure of merit increases with increase of $\mathrm{Si}$ concentration.

The values of all samples increase with increase of temperature. The maximum value is reached about $2 \times$

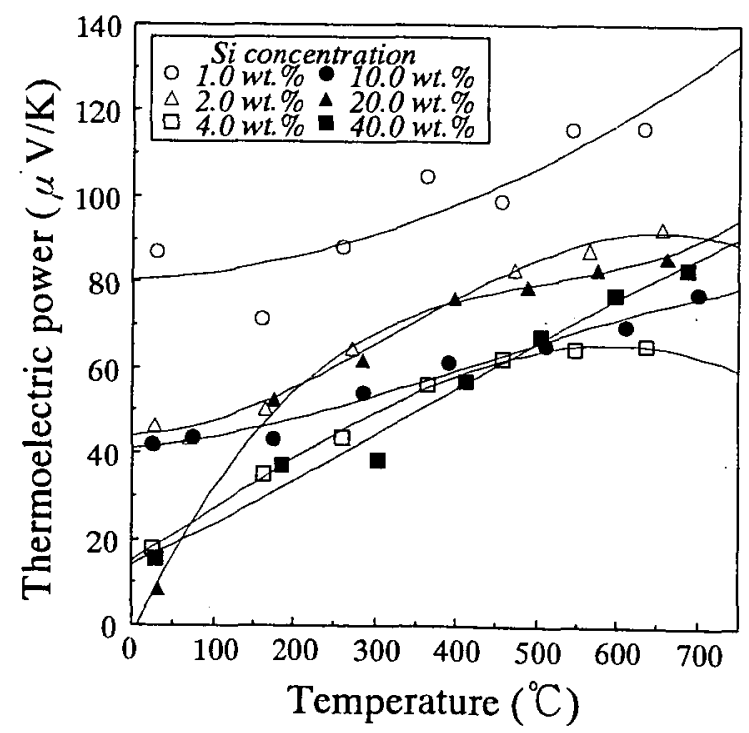

Fig.4 The temperature dependence of the thermoelectric power as a function of the Si concentration. 


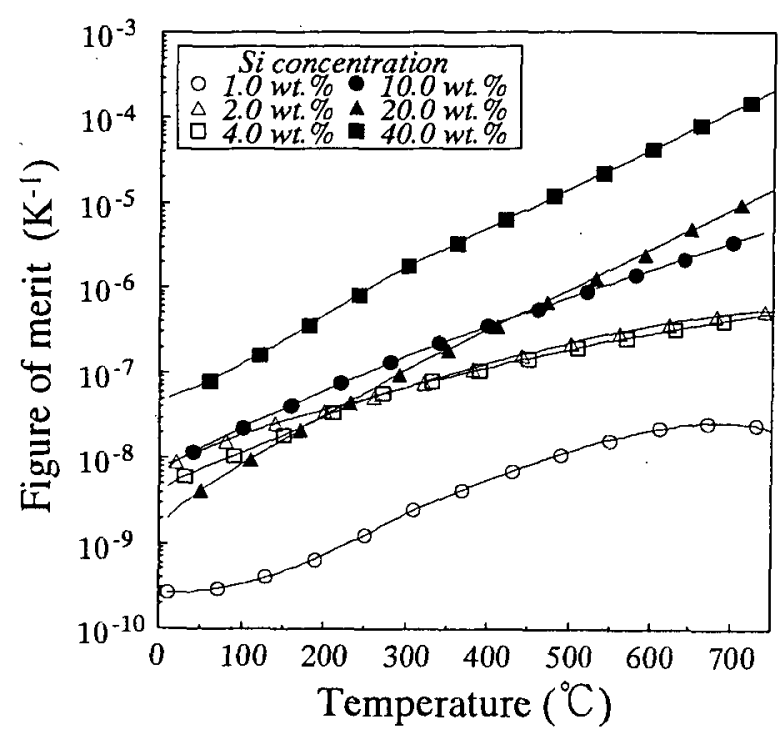

Fig.5 The temperature dependence of the figure of merit as a function of the Si concentration. In this figure, solid lines represent the results of calculation using the polynomials showed in the fig.2, fig. 3 and fig.4. Marks are used only for identification.

$10^{-4} \mathrm{~K}^{-1}$ at around $750{ }^{\circ} \mathrm{C}$, which is the one of the best value of the $\mathrm{SiC}$ based thermoelectric materials ${ }^{5-9}$. This large $\mathrm{Z}$ value is achieved by reduction of the thermal conductivity and electrical resistivity. The figure of merit of the higher concentration samples (10.0 wt.\%, $20.0 \mathrm{wt} . \%$ and $40.0 \mathrm{wt} . \%$ ) shows linear increase with temperature. On the other hand, the figure of merit of the lower concentration samples (1.0 wt.\%, $2.0 \mathrm{wt} . \%$ and $4.0 \mathrm{wt} . \%)$ shows weakly saturation tendency. Therefore we can conclude that the $\mathrm{SiC}$ system is a promising candidate for high temperature thermoelectric applications. It is challenging task to dope the additional impurity atoms to reduce the electrical resistivity with preserving the thermal conductivity value which is measured in this report.

\section{Conclusion}

The reduction of thermal conductivity is succeeded by excess addition of $\mathrm{Si}$. On the other hand, electrical resistivity and thermoelectric power exhibit the anomalous behavior which is almost independent of Si concentration. The figure of merit of the sample with $40.0 \mathrm{wt} . \% \mathrm{Si}$ concentration reaches about $2 \times 10^{-4} \mathrm{~K}^{-1}$ at around $750{ }^{\circ} \mathrm{C}$, that value is very close to the criterion for practical use.

\section{Reference}

1) T.Ohta, A.Yamamoto, T.Tanaka, T.Fujimaki and K.Kamisako: "Composition dependence on thermoelectric properties of undoped polycristal $\left(\mathrm{Bi}_{2} \mathrm{Te}_{3}\right)_{1-y}\left(\mathrm{Sb}_{2} \mathrm{Te}_{3}\right)_{y}$ prepared by PIES method", Proc. 14th Int. Conf. on Thermoelectrics (ICT'95), St. Petersburg, Russia, (1995)24.

2) M.Ohtaki, T.Tsubota, K.Eguchi and H.Arai: "Thermoelectric performance of $\mathrm{ZnO}$-based mixed oxides as a promising high-temperature material", Proc. 14th Int. Conf. on Thermoelectrics (ICT'95), St. Petersburg, Russia, (1995)245.

3) K.Okano: "SiC焼結体の電気伝導", Yogyo-Kyokai-Shi, 94(1986)219, (in Japanese).

4) Y.Takeda, K.Nakamura, K.Maeda and Y.Matusita: "SiC焼結体の熱伝導率及び電気抵抗率に及ほす燒結助剂 の影響 ", Yogyo-Kyokai-Shi, 95(1987)860, (in Japanese).

5) W.S.Seo, K.Watari and K.Koumoto: "Thermoelectric conversion efficiency of porous $\mathrm{SiC}$ improved by microstructure regulation", Proc. 12th Int. Conf. on Thermoelectrics (ICT'93), Yokohama, Japan, (1993)175.

6) Y.Okamoto, A.Aruga, H.Tashiro, J.Morimoto, T. Miyakawa and S.Fujimoto: "Temperature dependence of thermoelectric properties of SiC:Al", Proc. 14th Int. Conf. on Thermoelectrics (ICT'95), St. Petersburg, Russia, (1995)269.

7) K.Kato, S.Tanaka, Y.Okamoto and J.Morimoto: "Thermoelectric Properties of SiC/Ag System at room temperature ", Mem. Natl. Def. Acad., 37(1997)1.

8) Y.Okamoto, K.Kato, K.Asai, J.Morimoto and T. Miyakawa: "The characteristics of high temperature thermoelectric material: sintered $\mathrm{SiC} / \mathrm{Cu}$ ", submitted in J. Matr. Sci.

9) Y.Okamoto and K.Kato: "A study for Thermoelectric Properties of Ni Doped SiC Sintered Thermoelectric Semiconductor", Mem. Natl. Def. Acad., 37(1997)15.

10) H.Wada, M.Watanabe, J.Morimoto and T.Miyakawa: "Photopyroelectric (PPE) determination of thermal diffusivity of $\mathrm{Bi}_{2} \mathrm{Te}_{2.85} \mathrm{Se}_{0.15}$ sintered thermoelectric semiconductors", J. Mater. Res., 6(1991)1711.

11) J.Morimoto, Y.Okamoto and T.Miyakawa: "Thermal Diffusivity of Semiconductors Evaluated by Differential PPE Method", Jpn. J. Appl. Phys., 31 Suppl. 31-1(1992)38.

12) C.H.Pai, S.Takeda, H.Yanagida, W.S.Seo and K. Koumoto: "THERMOELECTRIC PROPERTIES OF POROUS SIC CERAMICS FABRICATED BY VARIOUS METHODS", 12th Int. Conf. on Thermoelectrics (ICT'93), Yokohama, Japan, (1993) 145 . 